ADP was similar between the two groups. In patients with high cardiovascular risk profiles early re-introduction of aspirin or other anti-platelet agents should be considered.

Competing interests None declared.

\section{PWE-162 SEASONAL AND DIURNAL VARIATION IN THE PRESENTATION AND SEVERITY OF ACUTE UPPER GASTROINTESTINAL BLEEDING}

doi:10.1136/gutjnl-2012-302514d.162

${ }^{1} \mathrm{~B}$ R Disney, ${ }^{*} \mathrm{R}$ Watson, ${ }^{2} \mathrm{~A}$ Blann, ${ }^{2} \mathrm{G}$ Lip, ${ }^{3} \mathrm{C}$ Tselepis, ${ }^{1} \mathrm{M}$ Anderson. ${ }^{1}$ Department of Gastroenterology, Sandwell and West Birmingham Hospitals NHS Trust, Birmingham, UK; ${ }^{2}$ Department of Cardiology, Sandwell and West Birmingham Hospitals NHS Trust, Birmingham, UK; ${ }^{3}$ Department of Cancer Sciences, University of Birmingham, Birmingham, UK

Introduction Acute upper gastrointestinal bleeding is a medical emergency associated with a significant health burden and risk of mortality. Previous studies have looked for diurnal and seasonal variations in presentation. No studies have addressed these issues in the UK population.

Methods All patients admitted with acute upper gastrointestinal bleeding to Sandwell and West Birmingham Hospitals NHS Trust from 1 January 2009 to 31 December 2009 were included in the study. Diurnal and seasonal differences in presentation were analysed using the $\chi^{2}$ test; differences in Rockall and Blatchford scores were analysed using the Kruskal-Wallis test followed by the Mann-Whitney $U$ test, with Bonferroni correction, to assess differences between individual groups.

Results Overall, 470 patients with acute upper gastrointestinal bleeding were admitted during the study period. Of these $67.2 \%$ were male and $32.8 \%$ female. The mean age of patients was $64.0 \pm 18.8$ years. Significant differences were seen in both diurnal and seasonal variation. Patients were more likely to present between the hours of 12:01-18:00 $(p<0.001)$. Admission rates were lower during the winter months $(p=0.028)$. The Rockall score showed significant diurnal variation $(p=0.048)$. No diurnal variation was seen in the Blatchford score $(p=0.39)$.

Conclusion Acute upper gastrointestinal bleeding shows a significant diurnal and seasonal variation in presentation. Diurnal variation is observed in Rockall scores, although this is of doubtful clinical relevance. The variation in presentation of acute upper gastrointestinal bleeding may have implications for the provision of endoscopy services.

Competing interests None declared.

\section{PWE-163 FACTORS PREDICTING EXTENDED LENGTH OF STAY FOLLOWING LAPAROSCOPIC NISSEN FUNDOPLICATION: A MOVE TOWARDS DAY CASE SURGERY}

doi:10.1136/gutjnl-2012-302514d.163

B Alkhaffaf, ${ }^{*}$ P Turner, R Date, M Mughal, J Ward, K Pursnani. Department of Oesophago-Gastric Surgery, Lancashire Teaching Hospitals NHS Foundation Trust, Preston, UK

Introduction There has been a move towards performing laparoscopic gastric fundoplication as a "day-case" procedure. This study aimed to determine which factors influence length of stay (LOS) to better enable patient selection for "day-case" fundoplication.

Methods This was a retrospective study of 229 consecutive laparoscopic Nissen fundoplications performed between 1999 and 2011. The primary outcome measure was length of hospital stay (LOS). Factors examined were patient age, main indication for surgery (large hiatus hernia or gastro-oesophageal reflux disease (GORD) and primary or redo surgery), history of previous surgery and presence and size of hiatus hernia.

Results Patients undergoing surgery for large hiatus herniae had an average 2-day greater LOS compared to those undergoing surgery for GORD $(p<0.001)$. Surgery for large hiatus herniae was also associated with a higher rate of conversion to open surgery (41\% vs $7 \% ; p<0.001)$. LOS was not affected by small or moderate sized hiatus herniae. A history of previous open upper abdominal surgery increased LOS by an average of 2 days $(p=0.010)$ and was associated with a higher rate of conversion to open surgery when compared to cases with no past surgical history (40\% vs $12.7 \%$; $p=0.036$ ). Redo fundoplication surgery led to an increased LOS by an average of 1.5 days $(p=0.044)$ when compared to primary surgery. There was a positive correlation between age and LOS $(\mathrm{p}=0.007)$.

Conclusion Factors which should exclude patients from undergoing "day-case" fundoplication include; large hiatus hernia as the main indication for intervention, redo surgery, a history of previous open upper abdominal surgery and advancing age.

Competing interests None declared.

\section{PWE-164 ASSESSMENT OF HELICOBACTER PYLORI IN IRON DEFICIENCY ANAEMIA: DO WE DO THIS?}

doi:10.1136/gutjnl-2012-302514d.164

C Daker, ${ }^{*}$ M Haji-Coll, N van Someren, K Besherdas. Department of Gastroenterology, Chase Farm Hospital, London, UK

Introduction Globally iron deficiency anaemia (IDA) is responsible for over two billion cases. In the western world, an estimated $2-5 \%$ of adult men and post menopausal woman suffer with IDA. GI loses account for many cases and patients will undergo endoscopy to exclude pathology here. Recent evidence suggests that infection with $H$ pylori (HP) should be considered despite the absence of peptic ulcer disease or other bleeding lesions in the GI tract. Hypothesised mechanisms for HP causing IDA are: chronic gastritis causing active bleeding, so iron is lost, achlorhydria decreasing iron absorption, the possibility that HP itself directly acquires iron so competing with the host, and also the possibility of an anaemia of chronic disease.

Methods The study aim was to determine whether patients with IDA referred for an gastroscopy (OGD) had a rapid urease test (CLO test) performed to confirm infection with HP. This was a single centre, retrospective analysis of consecutive patients endoscoped for IDA over 1 year upto January 2011. The endoscopy report was scrutinised for the performance of the CLO test and if performed the result was noted.

Results 194 Of 473 (41\%) patients undergoing OGD for IDA had their CLO test obtained. Of these 27 of 194 (5.7\%) were positive with 167 of 194 negative CLO tests.

Conclusion In this study, 59\% of patients endoscopied for IDA did not have a HP test. We may be missing a simple treatable cause for IDA in the upper GI tract within this group of patients. Of those who were not tested (270) 71 exhibited other significant pathology (angiodysplasia, gastric/colonic cancer, peptic ulcers). The association of IDA with HP is not universally recognised and we believe may be the reason as seen in this study not tested for in the absence of peptic ulcer disease or another cause for bleeding when endoscoping patients for IDA. We recommend routine testing (and eradication if detected) of HP in patients IDA undergoing OGD. In addition, testing and eradicating for HP may also reduce the risk of development of gastritis, peptic ulcer, MALToma and gastric cancer in those found to have the bacteria. 
Abstract PWE-164 Table 1

\begin{tabular}{lcc}
\hline CLO results & Totals & $\%$ \\
\hline Negative & 167 & 35.3 \\
Positive & 27 & 5.7 \\
Not done & 279 & 59 \\
\hline
\end{tabular}

Competing interests None declared

\section{PWE-165 CYP2C19*17 GAIN OF FUNCTION MUTATION IS ASSOCIATED WITH PEPTIC ULCER DISEASE}

doi:10.1136/gutjnl-2012-302514d.165

${ }^{1,2} \mathrm{C} 0$ Musumba, ${ }^{2} \mathrm{D}$ V Eker, ${ }^{3} \mathrm{~A}$ Jorgensen, ${ }^{1} \mathrm{D} \mathrm{M}$ Pritchard, ${ }^{2} \mathrm{M}$ Pirmohamed. ${ }^{1}$ Department of Gastroenterology, University of Liverpool, Liverpool, UK; ${ }^{2}$ Department of Molecular and Clinical Pharmacology, University of Liverpool, Liverpool, UK; ${ }^{3}$ Department of Biostatistics, University of Liverpool, Liverpool, UK

Introduction Studies show that single nucleotide polymorphisms (SNPs) in non-steroidal antiinflammatory drug (NSAID)-metabolising enzymes (mainly CYP2C9 and CYP2C8) may predispose NSAID-users to peptic ulcer disease (PUD) or upper gastrointestinal bleeding (UGIB), but results have been inconclusive.

Methods We hypothesised that the eight closely-linked clinically important SNPs in the CYP2C family of genes, namely CYP2C8*3 (rs11572080 and rs10509681), CYP2C8*4, CYP2C9*2, CYP2C9*3, CYP2C19*2, CYP2C19*3, and CYP2C19*17 predispose to PUD via impaired NSAID metabolism as well as other potentially important mechanisms (eg, metabolism of arachidonic acid (AA) and protonpump inhibitors-PPIs). Subjects diagnosed with PUD/UGIB at 13 hospitals in the UK between 2005 and 2011 were recruited and interviewed using a structured questionnaire, and categorised as either NSAID-users or non-users. UGIB was defined as haematemesis, melaena or anaemia, and endoscopic stigmata of recent bleeding. H pylori status was ascertained in most subjects. Following extraction of genomic DNA, genotyping was performed by KBioscience Ltd (UK). Logistic regression analysis was used to test for association between each SNP and risk of PUD/UGIB. Interaction terms were introduced to determine whether any observed genetic effects were influenced by factors including type of NSAID, PPI use and gender.

Results 1246 white patients were recruited and categorised as follows: 485 (39\%) PUD+/NSAID+; 357 (29\%) PUD+/NSAID-; $125(10 \%)$ PUD-/NSAID+; 280 (22\%) PUD-/NSAID-. Seven SNPs were included in the final analysis (CYP2C19*3 was monomorphic and excluded). All SNPs were in Hardy-Weinberg equilibrium. Logistic regression analysis of cases (PUD+; $n=842$ ) and controls (PUD-; $n=405$ ), assuming an additive mode of inheritance at each SNP, showed that only CYP2C19*17 was significantly associated with PUD ( $p=0.006)$, with suggestion of an allele-dose effect, even on classifying cases as those using only CYP2C9/ CYP2C8-substrate NSAIDs $(\mathrm{p}=0.005)$. Post-hoc analysis showed no interaction between CYP2C19*17 and NSAID type, PPI use or gender. Subgroup analysis per ulcer type showed CYP2C19*17 was significantly associated with gastric ulcers $(p=0.02)$, while only rs11572080 was associated with duodenal ulcers $(p=0.04)$. No SNPs were associated with UGIB.

Conclusion Possession of CYP2C19*17 allele is associated with PUD, especially gastric ulcers, regardless of aetiology. We postulate that this could be through its effect on the metabolism of AA or other endogenous substances, leading to impairment of gastrointestinal mucosal defences. Further studies are needed to correlate the functional consequences of CYP2C19*17 in the pathogenesis of PUD.
Competing interests None declared.

\section{PWE-166 INCREASING VACA TOXIN ACTIVITY ALTERS HELICOBACTER PYLORI COLONISATION DENSITY AND THE NATURE OF THE ACQUIRED IMMUNE RESPONSE IN A MOUSE MODEL OF INFECTION}

doi:10.1136/gutjnl-2012-302514d.166

D P Letley, * J Winter, A B Greenaway, K W Cook, J L Rhead, J C Atherton, $\mathrm{K}$ Robinson. Nottingham Digestive Diseases Centre Biomedical Research Unit, University of Nottingham, Nottingham, UK

Introduction Helicobacter pylori $(H p)$ infection causes chronic asymptomatic gastritis, and may lead to peptic ulceration and gastric cancer. Hp strains expressing more active forms of the vacuolating cytotoxin VacA are more strongly associated with disease than strains producing less toxigenic VacA. Hp infection stimulates a strong immunosuppressive interleukin-10 (IL-10) response, ${ }^{1}$ and VacA inhibits $T$ cell activation and interleukin-2 (IL2) production ${ }^{2}$ in vitro, but exactly how VacA contributes to disease development remains unclear.

Methods The vacA allelic type of the mouse-colonising $H p$ strain SS1 was found to be the non-toxigenic s $2 / \mathrm{i} 2 / \mathrm{m} 2$ form by PCRtyping. To assess whether vacuolating activity affects colonisation, a variant of strain SS1 expressing the more active s1/i1/m2 form of VacA (SS1/s1i1) was constructed. Vacuolating activities were compared in vitro by incubating RK13 epithelial cells with water extracts from each strain for $4 \mathrm{~h}$ in the presence of $10 \mathrm{mM}$ ammonium chloride, and counting the number of extensively vacuolated cells in random fields by light microscopy. C57BL/6 mice were infected with either SS1, SS1/s1i1 or given diluent as a placebo. After 3 weeks, gastric colonisation densities were assessed from weighed stomach tissue samples by quantitative culture on selective blood agar plates containing vancomycin, polymyxin $B$, bacitracin, nalidixic acid and amphotericin B. Immune responses were investigated by culturing spleen cells with and without $H p$ antigens in vitro and quantifying IL-2 and IL-10 concentrations in culture supernatants by ELISA.

Results As expected, the SS1 wild type strain induced virtually no vacuolation of RK13 cells (2\% of cells vacuolated). In contrast, its isogenic variant, SS1/s1i1, expressing the more active form of the toxin, induced vacuolation in $37 \%$ of cells $(p<0.01)$. Mutagenesis of SS1 vacA to a more active form had no effect on strain growth and viability in liquid culture media. Mice infected with SS1/s1i1 had approximately 100-fold lower colonisation densities compared to those administered the wild-type strain $(p<0.05)$. Splenocytes from mice infected with SS1/s1i1 secreted 2.8-fold lower IL-2 concentrations and 1.5-fold more IL-10 than cells from wildtype SS1-infected animals in response to stimulation with $H p$ antigens.

Conclusion Our data show that $H p$ strains expressing active VacA colonise less densely than less toxigenic strains, and that VacA modulates the immune response in vivo. We speculate that further characterisation of these effects will uncover why strains with active VacA are common in patients developing gastric cancer.

Competing interests None declared.

\section{REFERENCES}

1. Robinson K, Kenefeck R, Pidgeon EL, et al. Helicobacter pylori-induced peptic ulcer disease is associated with inadequate regulatory $T$ cell responses. Gut 2008; 57:1375-85

2. Gebert B, Fischer W, Weiss E, et al. Helicobacter pylori vacuolating cytotoxin inhibits T lymphocyte activation. Science 2003:301:1099-102. 